\title{
PSEUDO-MEASUREMENTS GENERATION USING ENERGY VALUES FROM SMART METERING DEVICES
}

\author{
Jorge ALVES \\ EDP Distribuição - Portugal \\ and Faculty of Engineering, University of Porto - Portugal \\ jorge.mendesalves@edp.pt
}

\author{
Jorge PEREIRA \\ INESC TEC - Portugal \\ and Faculty of Economics, University of Porto - Portugal \\ jpereira@inesctec.pt
}

\begin{abstract}
To enable the use of smart metering historical information of energy measurements in real time network operation, in this paper is proposed the generation of pseudo-measurements, which can be combined with real-time SCADA measurements and feed an online state estimation procedure. Hence increasing the network operator's situational awareness.

The goal is to obtain a better representation of the network operation points, voltage values, than the one that is possible to obtain with the direct use of smart metering data, which is based on average values, by increasing the amount of available real time data points.
\end{abstract}

\section{INTRODUCTION}

The electrical energy system revolution in Europe was started by the unbundling of transmission and distribution activities, from production and retail. This revolution started with the publication of the directive 96/92/CE approved in 19 December of 1996, by the European parliament. Both transmission and distribution were considered as natural monopolies subjected to regulatory supervision. At the same time electrical mobility and smart grid concepts were developed, and all around the world Distribution Systems Operators (DSO) launched pilot smart grid projects. Nowadays utilities are performing the roll out of those projects in a time of economy contraction and tighter regulatory framework, pushing DSO's to postpone grid investments and improve system efficiency.

Smart metering deployment is part of the smart grid roll out that can be independently implemented. In fact some DSO were already mandated by the regulatory agencies to deploy smart metering devices at all secondary substations. These devices will collect data from all secondary substation power transformers energy flows, enabling more detailed power flow studies and more accurate power losses assessment.

From the real time operation point of view no new real time measurements are available with this technology. Only historical data is available, and the existing measurements are not instant power measurements, but 15 minute period average power, of active and reactive energy flows.

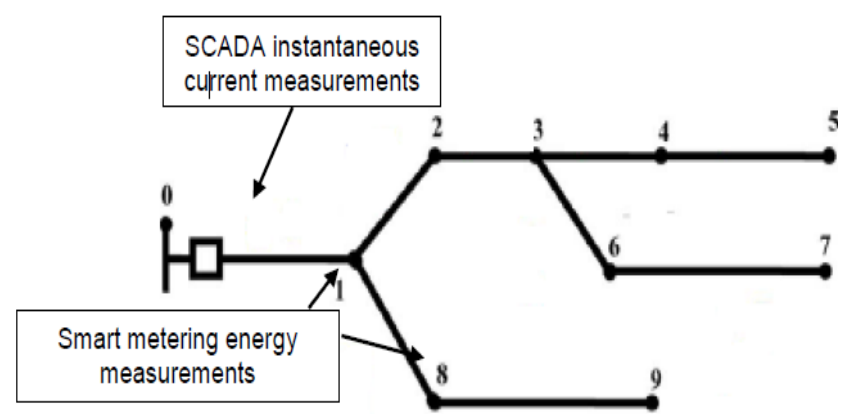

Figure 1 - Typical distribution network.

To enable the use of such information in real time network operation, at distribution network control centers, in this paper is proposed the use of smart metering information to generate instant power pseudomeasurements, that can be combined together with other SCADA real time measurements and running the state estimation procedure, to estimate the point of operation of the distribution network (Figure 1), hence increasing the network operators situational awareness.

The goal is to obtain a better representation of the network operation points, voltage magnitudes and phases values, than the one that is possible to obtain with the smart metering data by increasing the amount of available data points, and transform energy measurements (average values) into instantaneous power pseudo-measurements.

\section{PSEUDO-MEASUREMENTS GENERATION}

The method proposed to generate pseudo-measurements based on smart metering data, is founded on the Inverse Transform statistical method [1].

This method was applied considering the assumption that the probability density function of the average is the same as of the instantaneous values. So the generated data will have the same probability density function of the original data despite the latter being energy data and the first instantaneous power data.

The goal of the proposed method is to transform the constant average energy value, acquired for a period of 15 minutes, and obtain four instantaneous power measurements (Figure 2).

As already mentioned above the available data for each secondary substation is the 15 minute active and reactive energy average that circulates in the transformer of each secondary substation. 


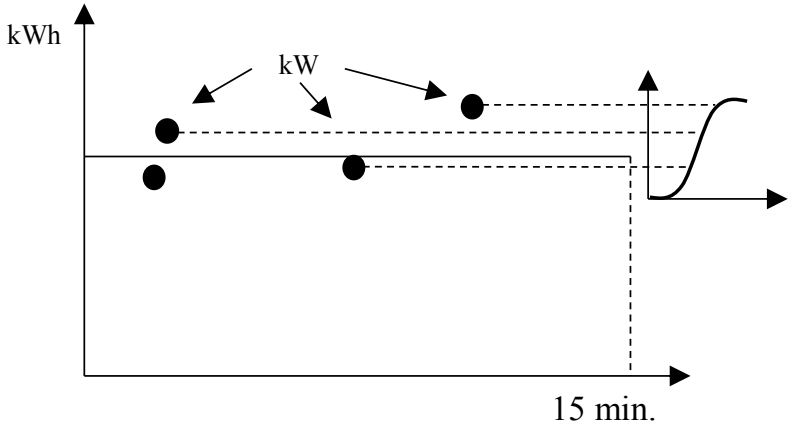

Figure 2 - Graphical representation of the pseudomeasurements generation, which corresponds to a transformation of an average energy value into four power measurements.

The Inverse Transform Method is based on a theorem that state: let $\mathrm{X}$ be a continuous random variable with distribution function $F_{X}$. Then $U=F_{X}(X)$ has uniform distribution $U_{[0 ; 1]}$. Or, let $U$ be a uniform random variable, $\mathrm{U} \sim \mathrm{U}_{[0 ; 1]}$. For any continuous distribution function $F_{X}$ the random variable $X$ defined by $\mathrm{X}=\mathrm{F}_{\mathrm{X}}{ }^{-1}(\mathrm{U})$ has distribution $\mathrm{F}_{\mathrm{X}}$.

The Inverse Transform Method algorithm is simple and it is depicted bellow:

1) Generate $U \sim U_{[0 ; 1]}$

2) Setting $X=F_{X}^{-1}(U)$, and $X$ has distribution function $\mathrm{F}_{\mathrm{X}}$

Where:

U - continuous variable with a uniform distribution between 0 and 1 ;

$\mathrm{X}$ - continuous variable with probability distribution function $\mathrm{F}_{\mathrm{X}}$;

$F_{X}$ - probability distribution function of variable $X$.

To test the proposed approach, a small real network was considered (Figure 3), where one year of smart metering data was collected for each of the three secondary substations (loads of nodes 2, 3 and 4).

The Inverse Transform Method was adapted in order to enable the generation of 4 pseudo-measurements for each available data.

The first step was therefore to transform the 15 minute period energy average into 15 minute power average values. Subsequently the Inverse Transform Method was separately applied to the 6 types of measurements available, active and reactive power injection at node 2 , 3 and 4. For each value of each type of variable, 4 pseudo-measurements were generated.

The generated data, which corresponds to 140176 data points for each variable, was then used as input for power flow studies. One power flow study was performed for each data point, and results of branch flows as well as node voltage magnitudes were collected

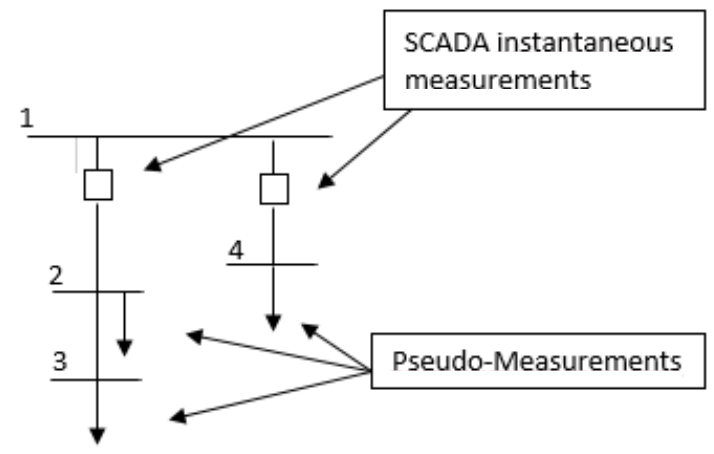

Figure 3 - Distribution test network.

Figure 4 show the empirical cumulative distribution function of the variable related to the active power consumption at node 2. From Figure 4 is possible to observe that the generated dataset match the empirical cumulative distribution function of the original data. This is the objective of the Inverse Transform Method. Noteworthy the generated dataset is 4 times bigger than the original dataset.

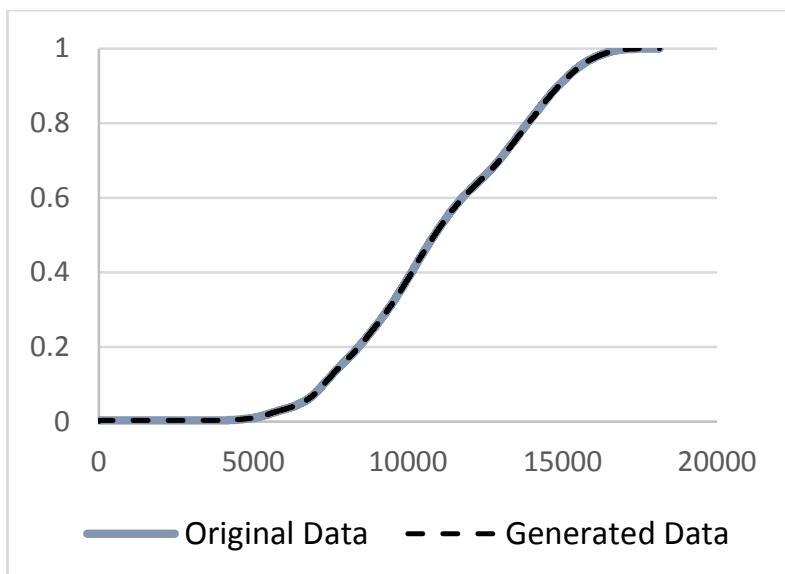

Figure 4 - Empirical cumulative distribution function of active power at node 2 .

\section{PSEUDO-MEASUREMENTS ASSESSMENT}

In order to assess the method a simple state estimation technique was implemented to compare the results obtained with the original data and the generated data. The goal of this step is solely to assess the pseudomeasurement generation procedure and not the estimation process.

The estimation process is based on a specific type of neural network, called autoencoder (Figure 5). This is a neural network trained so that the outputs are equal to the inputs, hence the number of neurons in the input and output layer is the same and typically hidden layers have smaller amount of neurons [2-4]. 


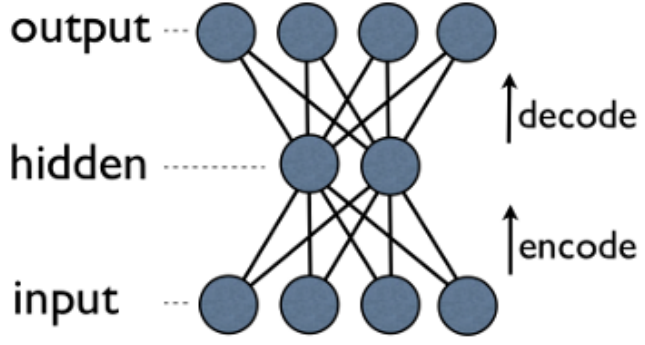

Figure 5 - Autoencoder.

The training procedure adopted was the extreme learning technique [5] which has very efficient execution times, when compared to the traditional back propagation neural network training method. In the training phase one expects that the autoencoder is able to learn the nonlinear relationships between the electrical variables.

The estimation process has the capability to combine real-time measurements with pseudo-measurements (non-real time telemetered measurements) due to the fact that in the estimation phase one combine the autoencoder input/output with an optimization algorithm, that for the non-telemetered data the algorithm will minimize the difference between the outputs and the inputs of the autoencoder. The optimization algorithm chosen was the Evolutionary Particle Swarm Optimization algorithm [6].

To test the pseudo-measurement generation developed technique, two neural networks of autoencoder type, one with the original dataset power flow results (35044 data points for each variable) and other with the generated data power flow results (140176 data points for each variable), were trained.

Apart from the 140176 generated data points, more 200 were generated to create the test dataset, and 200 power flow studies were performed. Then from this dataset the voltage magnitude at node 1 (V1), and the current magnitude in branches 1-2 (I1-2) and 1-4 (I1-4) were considered as real-time telemetered data (know values that characterized the operating point); and voltage magnitudes at nodes 2 (V2), 3 (V3) and 4 (V4), and the branch active and reactive power flows at branches 1-2 (P1-2 and Q1-2), 1-4 (P1-4 and Q1-4) and 2-3 (P2-3 and $\mathrm{Q} 2-3)$ were considered as non-telemetered values, hence to be estimated (Figure 3 ).

To summarize in the first set of telemetered/nontelemetered data, from 3 telemetered measurements, one estimates 9 non-telemetered measurements, in a total dimension of the solution space of 12 measurements.

The results presented in Table 1 and Table 2 support the hypothesis that the Inverse Transform Method is suitable to generate pseudo-measurements, and increase the representation of the network operation points. The ones obtained with the autoencoder trained with the generated data are in general better than the ones obtained with the autoencoder trained with the original data. In only one variable P1-4, active power in branch $1-4$, the results are not improved. On the other hand for a small network like the one presented in Figure 3, the percentage errors presented in Table 1 do not represent a good estimate for the system variables, as for the reactive power in branch 1-2, an average percentage error of $54 \%$ (autoencoder trained with the original data) and $41 \%$ (autoencoder trained with the generated data) are not acceptable.

Table 1 - Estimation results obtained for an autoencoder trained with original data (A1) and with generated data (A2); for the first set of telemetered/non-telemetered data.

\begin{tabular}{|c|c|c|}
\cline { 2 - 3 } \multicolumn{1}{c|}{} & A1 & A2 \\
\hline V1 & $0 \%$ & $0 \%$ \\
\hline I1-2 & $0 \%$ & $0 \%$ \\
\hline I1-4 & $0 \%$ & $0 \%$ \\
\hline V2 & $0 \%$ & $0 \%$ \\
\hline V3 & $0 \%$ & $0 \%$ \\
\hline V4 & $0 \%$ & $0 \%$ \\
\hline P2-3 & $28 \%$ & $22 \%$ \\
\hline Q2-3 & $34 \%$ & $29 \%$ \\
\hline P1-2 & $2 \%$ & $2 \%$ \\
\hline Q1-2 & $54 \%$ & $41 \%$ \\
\hline P1-4 & $13 \%$ & $35 \%$ \\
\hline Q1-4 & $43 \%$ & $27 \%$ \\
\hline
\end{tabular}

Table 2 - Estimation results obtained for an autoencoder trained with original data (A1) and with generated data (A2); for the second set of telemetered/non-telemetered data.

\begin{tabular}{|c|c|c|}
\cline { 2 - 3 } \multicolumn{1}{c|}{} & Original Data & Generated Data \\
\hline V1 & $0 \%$ & $0 \%$ \\
\hline I1-2 & $2 \%$ & $2 \%$ \\
\hline V2 & $21 \%$ & $21 \%$ \\
\hline V3 & $0 \%$ & $1 \%$ \\
\hline V4 & $0 \%$ & $1 \%$ \\
\hline P2-3 & $24 \%$ & $1 \%$ \\
\hline Q2-3 & $35 \%$ & $15 \%$ \\
\hline P1-2 & $0 \%$ & $28 \%$ \\
\hline Q1-2 & $0 \%$ & $0 \%$ \\
\hline P1-4 & $0 \%$ & $0 \%$ \\
\hline Q1-4 & $0 \%$ & $0 \%$ \\
\hline
\end{tabular}


These results suggest that the estimation errors can be reduced by adopting another set of real-time telemetered measurements. In the second set of telemetered/nontelemetered measurements, one considered as telemetered data the active and reactive power flows at branches 1-2 and 1-4, which represent 4 telemetered measurements.

Regarding the second set of telemetered/nontelemetered data the results (Table 2) showed a decrease of the estimation percentage average error, when compared with the results presented in Table 1 . Furthermore better results are obtained for the branch power flows, by the estimation performed with the autoencoder trained with the generated data, than the ones obtained with autoencoder trained using the original data. A reduction of $9 \%$ and $8 \%$ is attained for active and reactive power in branch $2-3$, respectively.

It is worth mention that a $0 \%$ error is achieved for the power flows in branches 1-2 and 1-4, due to the fact that in latter test these values are known (considered as realtime telemetered measurements).

\section{CONCLUSIONS}

The estimation results obtained suggest that the developed pseudo-measurements generation technique is suitable and efficient, as better results are achieved with the autoencoder trained with the generated data, when compared with the results obtained using the original data set to train the autoencoder. According to the results presented in Table 1 and Table 2, the estimation errors obtained with the autoencoder trained with the generated data, are smaller than the ones obtained with the autoencoder trained with the original data.

Combining this approach with an online state estimation technique, the network situational awareness at distribution network control centers can be greatly improved.

On the other hand, from the application of the proposed estimation technique is possible to conclude that this is a difficult problem due to the fact that the information contained in the voltage magnitude at the feeder busbar and the current magnitude at the beginning of the MV feeders, can be insufficient to characterize the feeder operation point, related to observability problems.

On top of this challenge, the fact that voltages at distribution levels are very much influenced by the presence of online voltage regulators, decreases the correlation between the voltage magnitude values at the feeder busbar and the reactive power flows along the feeder. This is the reason why estimation errors are greater in the reactive power flow branch estimation.

So for the same values of current and voltage magnitude different scenarios can occur, due to the presence of distributed generation and reactive power compensation. This turns the problem very difficult to solve with only real-time measurements of voltage and current magnitudes at the begging of the feeder.

\section{ACKNOWLEDGMENTS}

This work is financed by the ERDF - European Regional Development Fund through the Operational Programme for Competitiveness and Internationalisation - COMPETE 2020 Programme within project «POCI-01-0145-FEDER-006961», and by National Funds through the FCT - Fundação para a Ciência e a Tecnologia (Portuguese Foundation for Science and Technology) as part of project UID/EEA/50014/2013.

The first author Jorge Alves was financed by EDP Distribuição.

\section{REFERENCES}

[1] Sheldon Ross, 2013, Chapter 5 - Generating Continuous Random Variables, In Simulation (Fifth Edition), Academic Press, 69-96, ISBN 978-0-12415825-2.

[2] V. Miranda, J. Krstulovic, H. Keko, C. Moreira, and J. Pereira, 2012, "Reconstructing Missing Data in State Estimation With Autoencoders", IEEE Transactions on Power Systems, vol. 27, no. 2, 604-611.

[3] J. Krstulovic, V. Miranda, A. J. A. Simoes Costa, and J. Pereira, 2013, "Towards an AutoAssociative Topology State Estimator", IEEE Transactions on Power Systems, vol. 28, no. 3, 3311-3318.

[4] P. N. P. Barbeiro, H. Teixeira, J. Krstulovic, J. Pereira, and F. J. Soares, 2015, "Exploiting autoencoders for three-phase state estimation in unbalanced distributions grids", Electric Power Systems Research, vol. 123, 108-118.

[5] G.-B. Huang, Q.-Y. Zhu, and C.-K. Siew, 2006, "Extreme learning machine: Theory and applications", Neurocomputing, vol. 70, no. 1-3, 489-501

[6] V. Miranda and N. Fonseca, 2002, "EPSO -Best-oftwo-worlds Meta-heuristic Applied to Power System Problems," Proceedings of the IEEE Congress on Evolutionary Computation, 10801085. 